\title{
The Louisiana Space Consortium Student Sounding Balloon Program
}

\author{
M. Stewart ${ }^{1}$, D. Browne, B. Ellison, J. Giammanco, D. Granger, T. G. Guzik, J.P. Wefel \\ Louisiana State University, Baton Rouge, LA, 70803
}

Since the fall of 2003 the Louisiana Aerospace Catalyst Experiences for Students (LaACES) program has been providing university students a two semester project that culminates with the flight of a scientific balloon experiment. During the first semester students complete the Student Ballooning Course (SBC) which teaches basic skills necessary to develop a working scientific payload. The SBC consists of a series of lectures and activities providing instruction in electronics, programming, project management, balloon payload design, and introductory circuit assembly. The SBC introduces the BalloonSat, a sub-assembly designed at LSU for LaACES which contains a microcontroller, real-time clock and a four channel analog-to-digital converter. The second semester is spent on the design, development, testing and calibration of the payloads. Upon completion of the Flight Readiness Review, students travel to the NASA Columbia Scientific Balloon Facility (CSBF) in Palestine, Texas for integration, launch, recovery and science presentations. A flight capable Automatic Packet Reporting System (APRS) radio beacon armed with GPS and command capable cut down was developed to track the balloon during flight and to cut-down the payloads. Tracking vehicles are outfitted with radios tuned to APRS frequency and laptops displaying maps of the payload location. Here we describe LaACES; program development, tools and technologies, implementation, program, management issues and flight experiences.

\section{Introduction}

$\mathrm{T}$ The Louisiana Aerospace Catalyst Experiences for Students (LaACES) is a rigorous two semester program in which student participants from various institutions across Louisiana learn how to manage a real-world scientific experiment from the design phase to data analysis and results presentation ${ }^{14}$. The end result for each

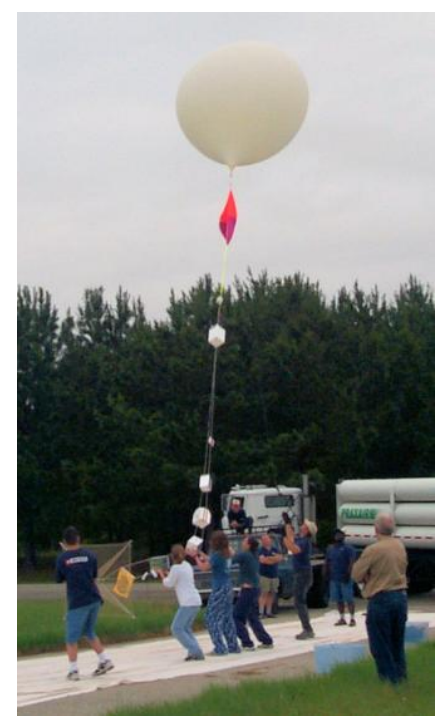

Figure 1. Launch of ACES-1 student group is a fully functional science experiment designed, developed and tested by students and flown to an altitude of approximately 100,000 feet with the goal of collecting 'good science' (Fig. 1). The target student is typically a sophomore enrolled in a Science, Technology, Engineering or Mathematics (STEM) related curriculum. Most students entering the program lack an understanding of electronics, micro-controller programming, program management and group dynamics; they also need to develop technical writing and presentation skills. A large portion of the first semester is dedicated to providing the students with the skills necessary to complete the project while the rest of the semester is dedicated to payload design. The Student Ballooning Course (SBC) was developed to provide these skills and includes a series of lectures, activities, design documents and presentations. During the second semester, students spend time bringing their designs to fruition. Students that complete the program gain "hands-on" practical experience working with a team to complete a project in a time-critical environment. They also learn a specific set of skills directly applicable to STEM related fields as well as improve upon their written and oral communication skills. Finally, the sense of accomplishment and the confidence built due to the completion of such a demanding program is immeasurable and it is the goal of LaACES 'to inspire' so that participants continue towards STEM related careers.

\footnotetext{
${ }^{1}$ All authors, Department of Physics \& Astronomy, 202 Nicholson Hall
} 


\title{
II. Historical Perspective
}

LaACES matured from the pilot program Aerospace Catalyst Experiences for Students (ACES) ${ }^{14}$ which was created by LaSPACE as a way to increase the number of STEM professionals within the United States (e.g. Fig. 2). This follows the Walker Report, which states that the "future of the U.S. Aerospace industry depends on the ability of the industry to attract, develop and retain a properly skilled professional, scientific, engineering and production workforce." Every two years the National Science Board (NSB) releases a report containing indicators denoting the status of STEM related fields. The NSB Chair, Warren Washington, made the following comments regarding the 2004 NSB report: "The U.S. is in a 'long-distance race' to retain its world leadership in science and technology. For many years we have benefited from minimal competition in the global S\&E labor market, but attractive and competitive alternatives are now expanding around the world, ... We must develop more fully our native talent." ${ }^{2,3}$ It is important to attract younger people to the STEM related fields because "the total number of retirements among S\&Edegreed workers will increase dramatically over the next 20 years. More than half of S\&E-degreed workers are aged 40 or older. ${ }^{3,4}$ STEM related fields are important for many reasons. One is they drive today's technology oriented economy. Science spawns innovation, breeding new technologies which in turn produce higher salary engineering and technical jobs available to those who have been properly trained. As described in a 2001 national security report,

\begin{abstract}
"In short, our problems in this area are becoming cumulative. The nation is on the verge of a downward spiral in which current shortages will beget even more acute future shortages of high-quality professionals and competent teachers. The word "crisis" is much overused, but it is entirely appropriate here. If the United States does not stop and reverse negative educational trends-the general teacher shortage, and the downward spiral in science and math education and performance-it will be unable to maintain its position of global leadership over the next quarter century."
\end{abstract}

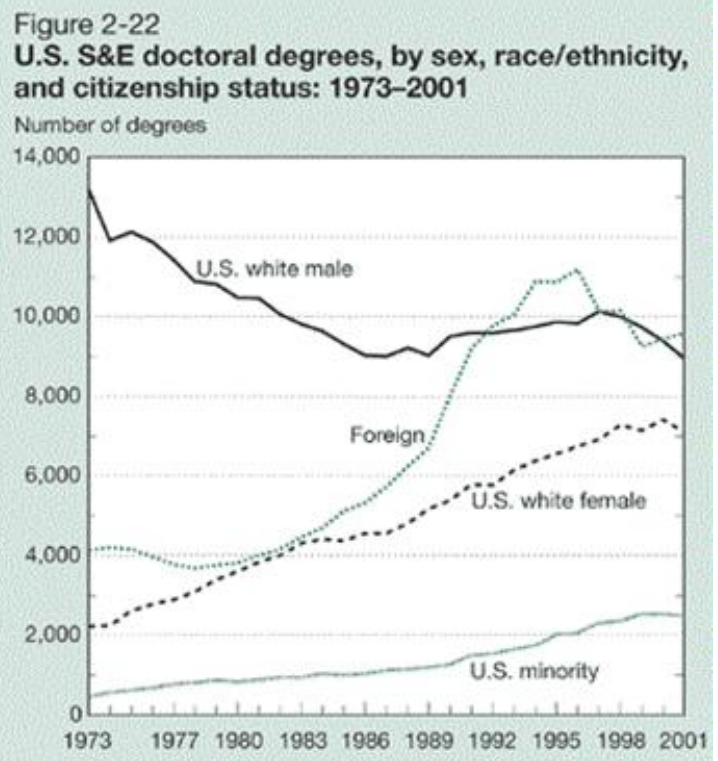

Figure 2. Overall trends in science and engineering doctoral degrees awarded ${ }^{3}$.

The primary goal of ACES was to attract and retain new students to STEM related fields utilizing an aerospace theme. ACES provided students with a background to develop and manage modern aerospace projects and gave students practical experience with sensors, electronics and "spacecraft" systems. These students were grouped into four teams of three to four students each and were exposed to the aerospace project development life cycle using the design, fabrication, testing and operation of small payloads (i.e. $<0.5$ kilogram) launched on a sounding balloon as a 'spacecraft simulation'. They were required to produce reports, establish schedules and milestones, and undergo reviews similar to the kinds of metrics used to track NASA projects. At the end of the academic year, following their Flight Readiness Review (FRR), the group traveled to NSBF in Palestine, Texas (Fig. 3) where their payloads were launched, operated during flight and recovered, and the data analyzed. With the success of the ACES pilot program

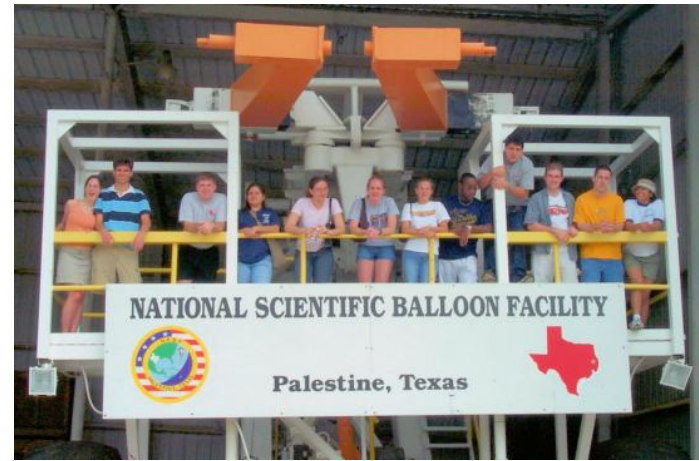

Figure 3. LaACES students posing for a picture on 'Tiny Tim' at the CSBF in Palestine, Texas.
-- three of the four groups produced results and science presentations -- NASA approved LaACES funding through Space Grant in early 2004. The experience gained in the ACES program indicated that a course with strict bounds should be developed and common hardware designed to ensure the smooth operation of the LACES project. The Student Ballooning Course (SBC) was designed in the spring and summer of 2004. It contains lessons and activities designed to interactively involve the participating students to prepare them to design, build and fly a science payload. The BalloonSat board was designed and made available to the student groups as a tool to teach electronics and micro-controller programming. LaACES involves student teams from institutions across Louisiana while the balloon support activities are centered at Louisiana State University.

$2^{\text {nd }}$ Annual Academic High Altitude Conference 


\section{The Student Ballooning Course}

The SBC is designed to take from August into December with two hour lectures given twice each week. The lectures consist of 33 PowerPoint presentations covering the primary topics relevant to the program ${ }^{14}$. There are also 30 descriptions of hands-on activities that complement the lectures and are critical in skill building. A complete list of materials as well as all the hardware necessary to complete all exercises is provided and includes all the components needed to build the SkeeterSat and BalloonSat boards. The lectures and activities are divided into five major units: electronics, programming, project management, balloon payload design and science. The electronics lectures provide the basic knowledge about circuits, sensor interfacing and data acquisition. The programming lectures teach the students how to direct the micro-controller to read and store data, and interface to the other devices needed for the data acquisition systems. The project management sessions focus on how to plan, manage and track the progress of a project while the balloon payload design section provides the facts and skills relevant to the successful development of a payload for the 'near space' environment. The lectures are taught by five instructors: one dedicated to project management, another to programming, two to electronics and one to developing writing and presentation skills.

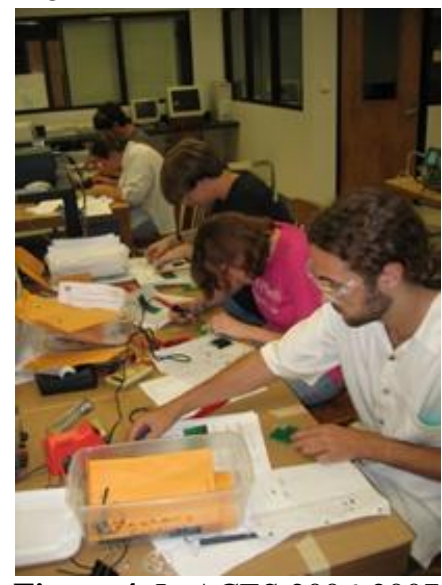

Figure 4. LaACES 2006-2007 students busy in the lab.

\section{A. The Learning Curve: Lessons, Activities, Documentation \& Presentations}

There are seven electronics lectures, each accompanied by an activity designed to reinforce the lecture. The first electronic lectures introduce the students to various common electronic components, measurements made and the instruments used to make them plus soldering and assembly techniques. These first few electronic lectures are reinforced by the SkeeterSat activity. The

"SkeeterSat (Fig. 5) is a simple data collection system that presents its data as audio tones. If connected to an inexpensive Family Radio Service (FRS) handheld "walkie-talkie", it forms a rudimentary radio telemetry system. Temperature and light intensity are the two physical parameters monitored. The frequency (pitch) of the audible beep produced by SkeeterSat depends on the ambient temperature. The time interval between beeps depends upon the intensity of light falling on the unit. Computer software (Spectrogram) can be used to convert the tone frequency and interval into numerical values that can be calibrated to produce the actual temperature and light intensity."

The students use Spectrogram to calibrate the SkeeterSat and write the first of many reports all of which are critiqued.

At this point the students should be comfortable with reading rudimentary schematics and assembling circuit boards, so introductory programming lectures and activities are initiated. The programming lectures start with a description of each of the components on the BalloonSat board and have the students build one to use for the programming activities. The first programming lectures are dedicated to introducing the students to the microcontroller, specifically the BASIC Stamp micro-controller manufactured by Parallax and the PBASIC syntax, the language developed by Parallax to control the BASIC Stamp. The students are introduced to the PBASIC editor which communicates to the BASIC Stamp via an RS-232 connection between the PC running the PBASIC editor and the BASIC Stamp. Since most of the students have little programming experience, we introduce topics such as hexadecimal and binary numbering systems, binary coded decimal, and ASCII bit representation. Students are shown how to make use of the PBASIC command reference (Fig. 6) as well as datasheets for electronic components and other reference materials.

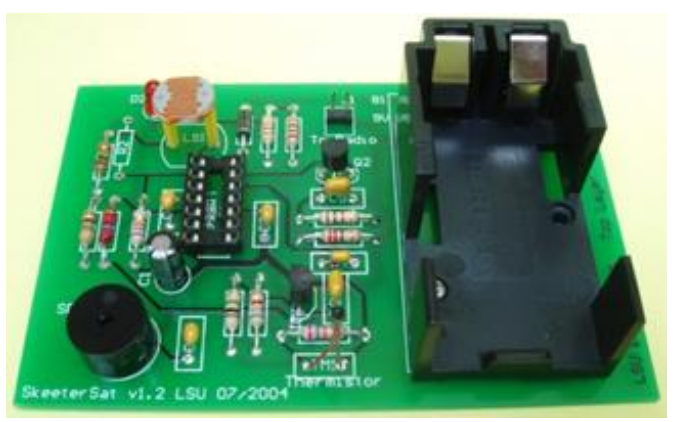

Figure 5. A fully assembled SkeeterSat.
With a grasp of electronics and programming, the next lectures and activities teach how to couple hardware and software to produce a system capable of completing tasks. Topics such as digital I/O signals and how to use them, modifying the offset and range of analog signals and analog-to-digital converters are covered. Other topics covered include serial I/O communication, specifically the two types used to communicate with the BalloonSat peripherals; serial peripheral interface (SPI) and inter-integrated circuit $\left(\mathrm{I}^{2} \mathrm{C}\right)$; interfacing to a real-time clock; and storing data to an external memory chip. The final software activity brings all the various components of the BalloonSat and knowledge gained from the beginning of the program together. The activity calls for the 
construction of a circuit to measure temperature using a diode whose output signal is between 450 and 800 $\mathrm{mV}$. The signal has to be shifted to 0 to 3 volts and delivered to the ADC on-board the BalloonSat. A program written by the students and run on the BASIC Stamp should start the conversion on the ADC, retrieve the digitized value, collect a timestamp from the real-time clock, and store the timestamp and digitized sensor value to the external memory chip. A report is written which describes the design and performance of the interface circuit and includes a narrative description of the procedure, tables of measured data, electronic circuit diagrams, a flow chart of the software and the PBASIC source code. This report marks a considerable achievement as it concludes the electronics and programming sub-topics.

With a simple data acquisition system built, the

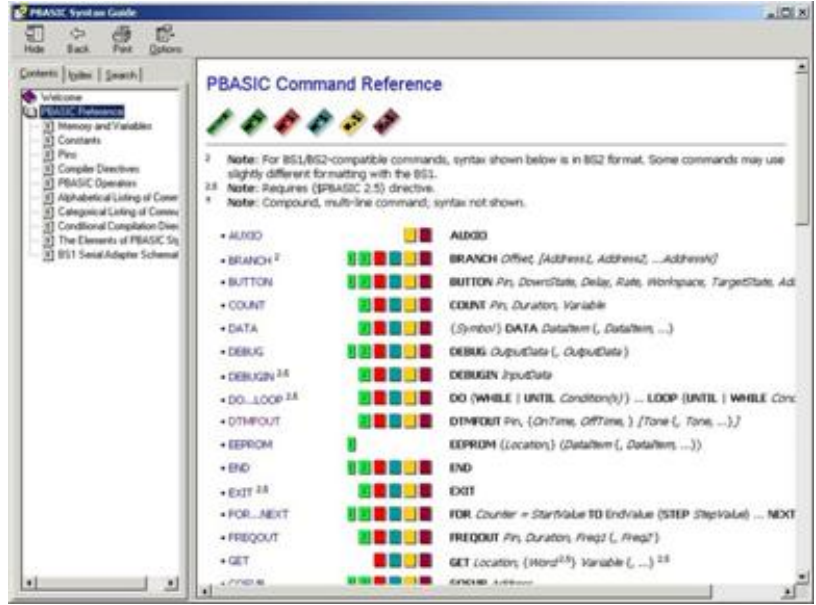

Figure 6.The BASIC Stamp Editor Reference manual students have to repeat the process and design a full-scale experiment within a group for a payload that will have to endure the extremes of high altitude ballooning while accurately documenting the process. To learn how to do this, students participate in lectures and activities which cover topics such as: project management, scientific writing, payload construction, system design, project scheduling, risk management, thermal design, system testing and debugging. The first lecture deals with scientific ballooning and covers the various types of experiments well suited towards high altitude ballooning; the environment the payload will experience, components of the balloon vehicle such as the sounding balloon, parachute, beacons and antennas, and typical flight profiles (Fig. 7). The next lecture and activity deal with project management and documentation where groups develop their team contracts which have been found to be vital for group success. "In this activity the students will begin thinking about project structure and management in the context of several real-world activities. The student groups need to understand the strengths and weaknesses of all team members, be able to construct an orderly sequence of events and tasks for a project and assign roles for team members within the project."7 Each group must present documentation throughout the program as writing skill development is an important goal of the LaACES program. From experience, this is an area in which students need a large amount of help. After the first few years of the LaACES program, an instructor was added to the group to concentrate on helping with documentation and presentation. This addition has made a positive difference in the students' reports. The next lectures concentrate on the design and construction of the payload boxes, including how to lay out a payload design using CAD software as well as the preferred materials for construction. The thermal lecture teaches the importance of pre-flight thermal analysis since components will fail if exposed to extreme temperatures. The thermal activity has the students calculate the expected temperature inside a payload box and then compare the calculated values with measured results. In this activity, the payload box contains a heat source consisting of a 1 watt light bulb and is placed in a large ice chest filled with dry ice. The calculations and measurements are done with and without a layer of insulation. The expected outcomes are: a quantitative feeling for heat flow, how the steady-state thermal equation is applied, understanding the temperature extremes that a balloon payload will experience during flight, the implications for choosing appropriate components and establishing procedures for thermal testing. The last lectures cover project tasking, costing and scheduling, and

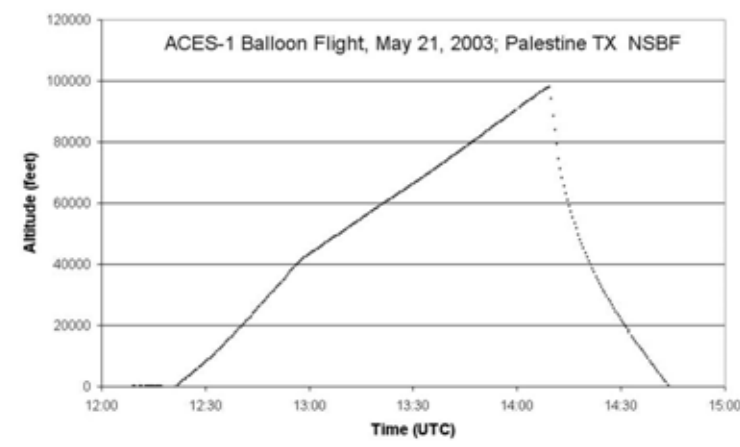

Figure 7. The flight profile of the first ACES balloon flight in terms of time versus altitude. mitigating risk factors.

The teams ultimately present two formal, controlled documents which are signed by each team member. They are the Critical Design Review (CDR) and the Flight Readiness Review (FRR). The CDR is written in stages and all LaACES instructors provide comments upon the completion of each stage. The CDR contains twelve sections discussing these topics: goals, objectives, requirements, science and technical background, payload design, development, and construction planning as well as mission operations, project management, scheduling and budget. The first stage of the CDR document is the Pre-Preliminary Design Review or Pre-PDR. At this stage the focus is on the team's goals, objectives and requirements, as well as a master schedule complete with a

$2^{\text {nd }}$ Annual Academic High Altitude Conference 
work breakdown structure, staffing plans and a timeline with milestones. Science and technical background applicable to the team's project make up a large portion of the Pre-PDR. The next stage is the Preliminary Design Review or PDR. At this stage the student teams are required to make corrections to the Pre-PDR, incorporate them into the PDR and add sections which detail payload design and development as well as project management. The next step toward the development of the CDR is the Pre-CDR. The teams correct for all comments made in the PDR and add a section describing risk management and the team's contingency plans to mitigate any known risks. To produce the CDR, any issues with the PreCDR are corrected and sections describing payload construction, mission operations and budgetary issues are added. The CDR is a stand-alone document such that one group

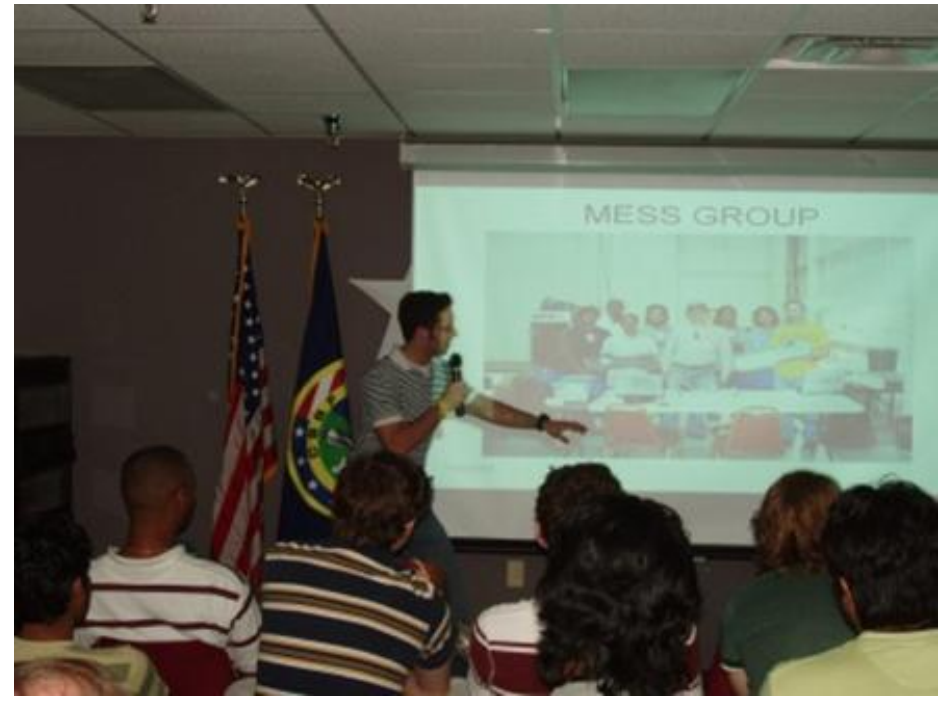

Figure 8. 2007-2008 LaACES students delivering their science presentations at CSBF.

should have no trouble building another team's payload by simply following the other's CDR The teams are also required to deliver presentations at the completion of each stage.

Upon completion of the payload build phase, the CDR is evolved into the FRR. In the FRR, all CDR issues are resolved, experiment readiness is documented, environmental test results are presented as proof that the payload meets flight requirements and descriptions for checkout and integration procedures are given.

\section{B. The Hardware: BalloonSat \& Sensor Boards}

The BalloonSat board (Fig. 9) evolved from the CanSat board developed by Professor Bob Twiggs at Stanford University's Space Science Development Laboratory. The BalloonSat incorporates the BASIC Stamp microcontroller an EEPROM for data storage and retrieval, a four channel, 8 bit analog-to-digital converter (ADC), a realtime clock with provision for battery backup and a prototyping area. This design is suitable for the laboratory activities as well as payload applications. The BalloonSat also features a voltage reference chip with a built in temperature sensor that can be jumpered into channel three of the ADC. The SBC kit comes with a 64 kilo-bit $\mathrm{I}^{2} \mathrm{C}$ EEPROM but can be swapped out with a pin compatible version with up to 512 kilo-bit of memory. The real-time clock has a SPI interface, registers for year, month, day, hour, minutes and seconds and includes on-chip 31 x 8 bit RAM scratchpad data storage. An RS-232 device can be connected to the BalloonSat serial port but level conversion might be necessary. A BASIC Stamp 2p 24 pin module was chosen as the microprocessor and with a processor speed of $20 \mathrm{MHz}$ it can execute approximately 12,000 PBASIC instructions per second. The BASIC Stamp has 32 bytes of RAM, 26 bytes for variables and 6 bytes for input, output and direction control of 16 general purpose I/O pins. There are also 128 bytes of scratchpad RAM and an 8 X 2 kilobyte EEPROM used primarily to store the programing instructions in up to 8 different 2 kilobyte slots. The BalloonSat is programmed using the BASIC Stamp

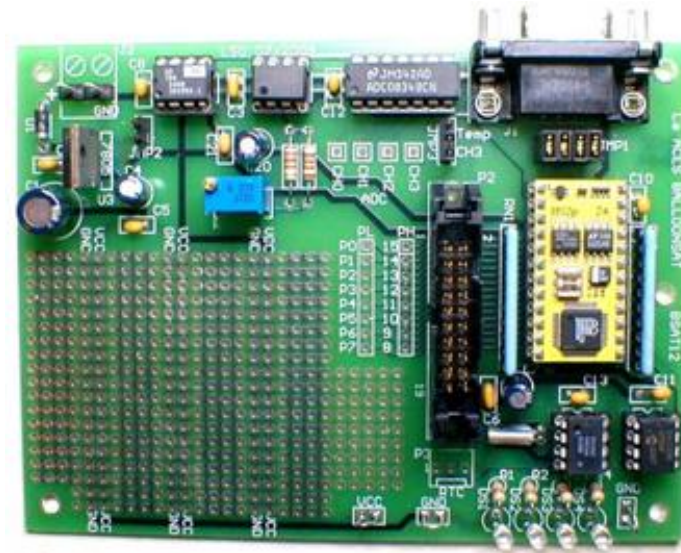

Figure 9. A fully assembled BalloonSat board. Editor which connects to the BASIC Stamp across a 9-pin RS232 connection at 9600 baud and can be installed on to the Windows XP, Windows 7or Mac OS platforms. PBASIC is the language used to program the Stamp. It does not take long for a student with little to no programming experience, with the help of an instructor, to develop a simple data acquisition system using the BalloonSat board. However, it can also be quite powerful: controlling servos, counting pulses, communicating with daughter boards using the built in instructions for $\mathrm{I}^{2} \mathrm{C}$, SPI and RS-232 communications. The BalloonSat includes a prototyping area for additional components and circuitry plus connection points for access to digital and analog interface signals. This area is typically used to add signal conditioning circuits for sensors defined by the particular mission's scientific and technical requirements. Student teams in past years have 
designed and implemented interfaces to sensors for studying ozone concentration, cosmic ray flux, ultra-violet radiation, magnetic field and atmospheric temperature, pressure and humidity studies.

Recently a custom sensor board was designed to provide the signal conditioning for a "standard" payload for measuring pressure, temperature and humidity (Figure 10). The sensor board conditions the sensor signals to conform to the 0-3 volt range of the BalloonSat ADC. An ICS1210 or ICS1230 piezoresistive pressure sensor is mounted on the board, and driven with a constant current derived from an LM234 current source. A difference amplifier with a voltage gain of approximately 30 produces an output of 0 to 3 volts for a pressure range of 0 to $15 \mathrm{psi}$. A gain adjustment potentiometer is provided for use in calibration.

The temperature sensor circuit uses an ordinary PN junction

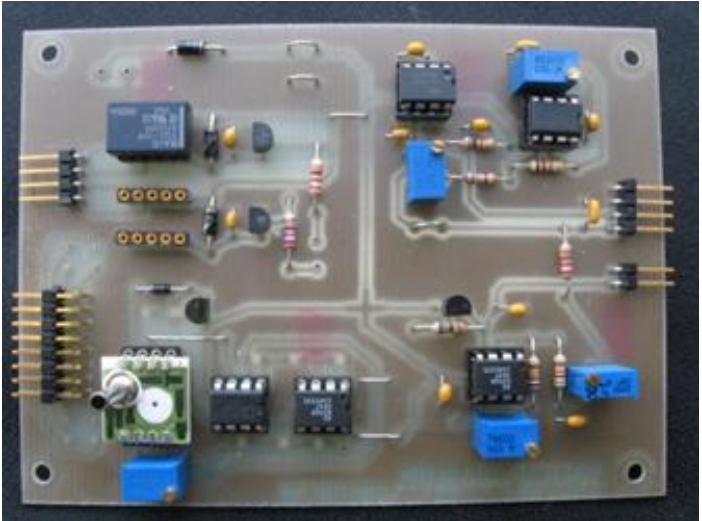

Figure 10. Pressure, temperature and humidity sensor board. diode (e.g. 1N457) as the sensor. A forward biased 1N457 exhibits a negative temperature coefficient of approximately $-2.5 \mathrm{mV} /$ Kelvin. In operation, an LM234 provides a constant current of $1 \mathrm{~mA}$ through the diode. The voltage across the diode varies over a range of about 240 to $300 \mathrm{mV}$ with the temperatures encountered (-80 to $+30 \mathrm{C}$ ). The signal conditioning circuit provides the gain and offset to produce the required 0 to $3 \mathrm{~V}$ output.

A stable, temperature-compensated reference voltage is provided from an Analog Devices REF-02. In addition, two relays are provided for controlling peripherals such as heaters or cameras. The current version of the BalloonSat printed circuit board includes a 26-pin interface connector to facilitate connection to the sensor board. The interface signals include inputs to the BalloonSat's ADC, the $\mathrm{I}^{2} \mathrm{C}$ clock and data lines, and several of the BASIC Stamp's programmable input/output pins. Using this standard interface reduces the time spent designing and building hardware while allowing more time to be allocated to the critical phases of calibration and system testing.

\section{Putting It All Together}

During the second semester of the program the student groups finish their CDR documents and begin building their experiments. Since the CDR document details every aspect of the build and test phases, the building cannot begin until the CDR is complete. The BalloonSat boards built during the lecture and activity sessions are used as the flight boards therefore it is not necessary that the students re-build them. They do however have to build their flight sensor boards which were prototyped during the design phase. Once the payload box is built, the BalloonSat and sensor boards can be mounted in the payload box and prepared for calibrations and system testing (Fig. 11). Flight and system calibration testing software are written from detailed software flowcharts presented in the CDR.

Temperature calibrations are performed by immersing the temperature sensor in an antifreeze / water mixture and coupled with a known calibrated sensor. The canister of antifreeze is placed in a cooler of dry ice. The temperature and $\mathrm{ADC}$ values are recorded as the temperature drops. Because the temperature senor signal is fed into one of the ADC channels on the BalloonSat it can be read and displayed by the calibration software. The ADC values (0-255 for an 8-bit ADC) are recorded with the temperature from the calibrated sensor. The temperature ranges from $25^{\circ}$ to $-50^{\circ}$ Celsius and are fed into an analysis program and plotted in order to find the best fit to the data. The relationship between temperature and voltage is linear in the PN junction diode used as the temperature sensor and therefore the calibration curve is linear as well (Fig. 12).
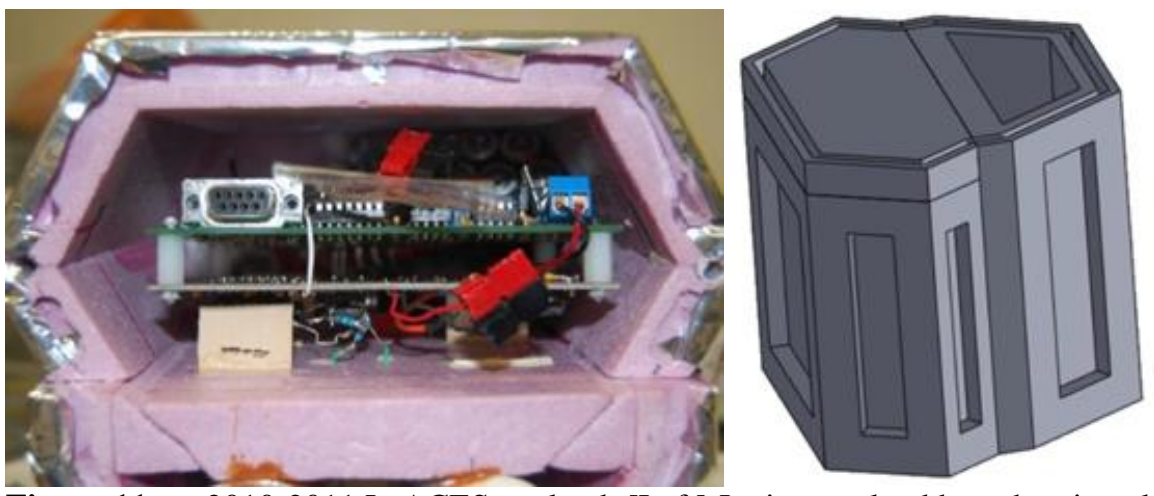

Figure 11. 2010-2011 LaACES payload: [Left] Jupiter payload box showing close up of electronics mounting; [Right] CAD of the Jupiter payload box ${ }^{8}$. 


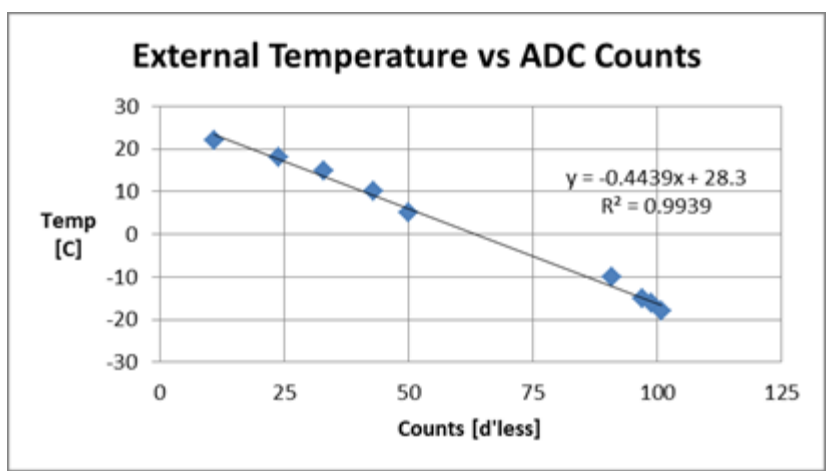

Figure 12. Example of temperature calibration results.

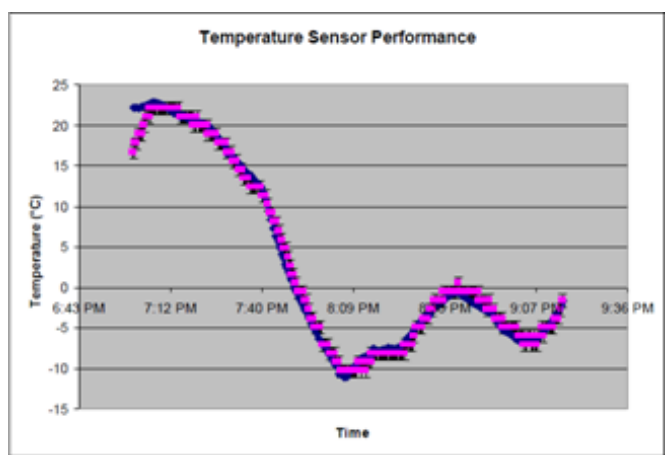

Figure 13. 2009-2010 LaACES team (Cosmic Ray) temperature system test results ${ }^{9}$

The pressure calibration is done in a similar fashion. The payload box is placed into a small vacuum chamber and a serial cable is fed through a hermetically sealed feed-through. The serial cable is run to the computer running the BASIC Stamp Editor in order to monitor the output of the calibration software. The pressure sensor signal is fed into a channel on the ADC and its digitized values are displayed on the screen at some time interval. Also, a known calibrated gauge measuring the absolute pressure inside the chamber is clearly visible so that its value can be recorded along with the ADC value. The vacuum pump is started and as the chamber decreases in pressure, readings from the calibrated gauge and the ADC values are recorded. Once the chamber is as close to zero atmospheres as possible, the values can be loaded into a plotting program and the data can be fitted. Again the pressure sensor used experiences a linear change relative to its surrounding pressure and therefore the calibration curve is linear.

Once calibrations are completed the student groups begin flight certification system tests of their payloads. The payloads should be in a flight-ready state so the tests are a good measurement of how their payloads will respond during flight. These tests should include an extreme temperature test where the payloads are subjected to temperatures ranging from $30^{\circ}$ to $-70^{\circ}$ Celsius, a vacuum test where the payloads will be subjected to decreasing and then increasing pressures intended to mimic flight conditions and a shock test where the payload is subjected to a $10 \mathrm{~g}$ force like one which may be experienced during flight. The student groups have to prove that their payloads are fully operational, collect data and behave nominally throughout each test in order to qualify for flight.

Flight certification is important because it could catch poor or improper payload performance allowing time for a student group to make repairs. As shown in Fig. 13, the team Cosmic Ray (2009-2010 LaACES campaign) payload performed extremely well during temperature system testing. The blue tracks their temperature sensor while the red tracks an independent measurement. They placed their payload in a refrigerator for 17 minutes then moved it to the freezer for 18 minutes and then into a cooler full of dry ice for 25 minutes and back to the freezer for 18 minutes then to the refrigerator again for 17 minutes and the interior temperature never dropped below $-10^{\circ}$ Celsius. However, they discovered that their detectors displayed some odd coincidence count results as the pressure neared 0 $\mathrm{mmHg}$ during the pressure system testing. This was determined to be due to arcing in their photo-multiplier tubes at very low pressures. The problem was found and fixed allowing them to fly their payload (Fig. 14). A good example of another LaACES student payload is PHAT-TACO presented at this conference. ${ }^{17}$

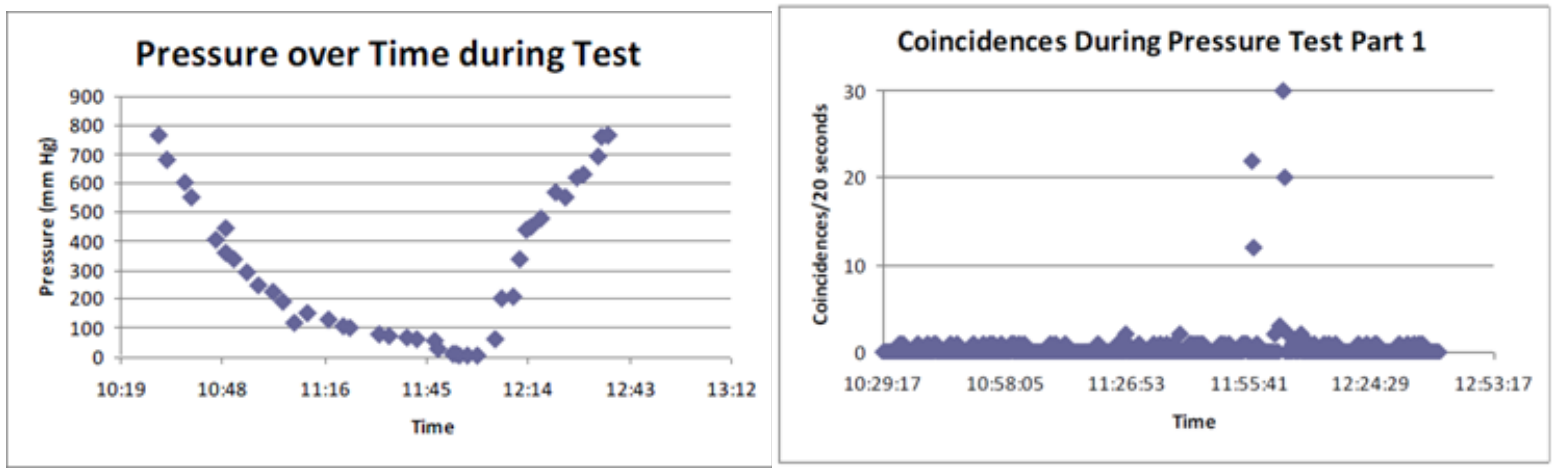

Figure 14. 2010-2011 LaACES team (Cosmic Ray) [top] pressure system test results, [bottom] detector coincidence results ${ }^{9}$ 


\section{Launch, Chase, Recovery \& Analysis}

The students who complete the LaACES program pack their payloads and equipment to travel to Palestine, Texas with the LaACES instructors and local ham operators for launch. Palestine, Texas is the home of the Columbia Scientific Balloon Facility (CSBF). The CSBF is operated under contract from NASA through New Mexico State University and is responsible for all large scale, U.S. scientific balloon launches. CSBF launches balloons from their home base in Palestine, from Antarctica, Sweden, Australia and Ft. Sumner, New Mexico. For LaACES, they let us use their facilities to prepare for launch and provide the helium for our balloons.

\section{A. Preparation and Launch}

The LaACES group arrives in Palestine on Sunday evening after a six hour drive from Baton Rouge. Work begins promptly at 8:30 am on Monday, at which time each student group delivers their FRR presentation. Once completed, the students have the rest of the day to make any last minute fixes and adjustments to their payloads. In order to fly, the payloads must be on the flight string by Monday evening. Launch is scheduled for the next day, and work starts before sunrise. Instructors and students arrive to the balloon base at $5 \mathrm{am}$ and student groups make final flight preparations to their payloads. The CSBF crew sets up the helium truck as the students and instructors walk the flight string complete with the payloads and beacons attached out to the launch pad. Inflation and final launch preparation takes less than an hour. Once conditions are right, the balloon is launched. Based on conditions, one of two launch methods is used. The first method used if winds are calm involves walking the flight train up until the designated launcher is holding the bottom of the flight train. A countdown is performed and the train is released. If surface conditions are breezy, the designated launcher holds the throat of the balloon and a representative from each group hold their payload so that each can be lifted without being impeded. Again a countdown is performed and the balloon is released so that it pulls the payloads out of

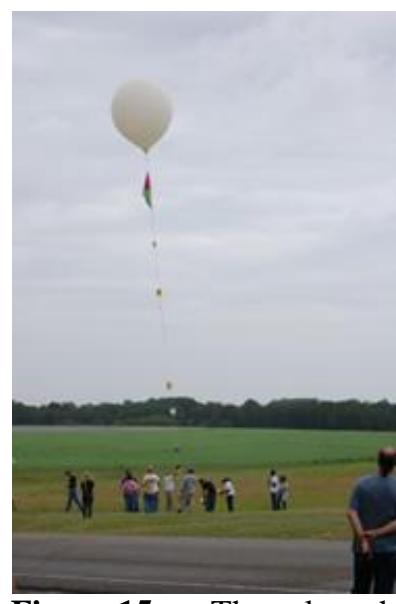

Figure 15. The launch of ACES-12 the hands as it rises (Fig. 15).

\section{B. The Chase: Tracking the Balloon \& Recovery}

Shortly after the balloon is launched, the chase vehicles set out. A system was designed for the LaACES project to track the balloon and issue the cut down command at the appropriate time. This system includes a series of radios, GPS units, a cut down mechanism on the flight train, and two or more vehicles each outfitted with a VHF transceiver. All vehicle radios are equipped with Automatic Position Reporting System (APRS) and a Terminal Node Controller (TNC) for reporting their location and receiving balloon position information. Each vehicle is also outfitted with a laptop which is connected to a GPS unit as well as to the radio. The laptop runs mapping software (Street Atlas) which uses the APRS data to track the balloon and to track all vehicles involved in the chase. The radios on the flight train are also configured with APRS and TNC capabilities and report its position. This system allows the vehicles to stay within line of sight of the balloon so that the cut down command can be issued once the balloon approaches 100,000 feet. Upon receipt of a valid cut down signal the on-board flight controller energizes a Ni-Chrome heater coil that melts the cord attaching the balloon to the parachute. The payloads, beacons and parachute fall away from the still rising balloon.

The ascent rate is approximately 1000 feet per minute so it takes the balloon less than an hour and a half to reach altitude. The descent rate is faster at 1400 feet per minute which puts the flight train on the ground, in a tree or in a pond about 40 minutes after cut down. The tracking team is usually close to the landing site within minutes of touchdown and locates the payloads and beacons shortly afterward, thanks to the beepers within the beacons. Retrieving the payloads is another matter. For some flights the payloads have landed in easily accessible locations, while others have landed high in trees in the middle of the woods on privately owned land. An array of recovery tools is brought to assist with difficult recoveries. Some of the tools include gloves, machetes, extending poles with various attachments, a chainsaw, a slingshot, a fishing pole and reel with plenty of fishing line, and orange 'do not accidentally shoot us' recovery vests.

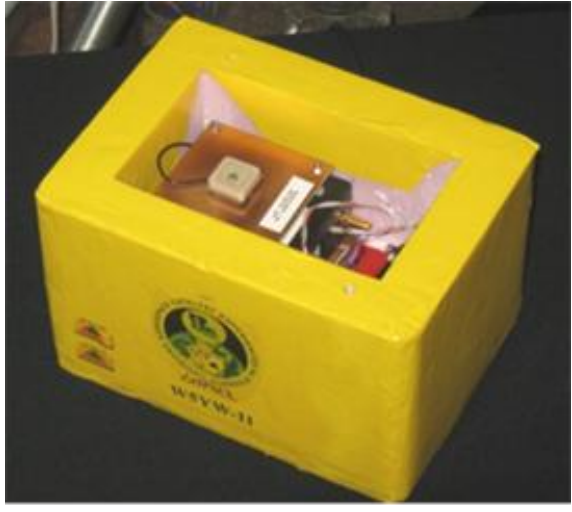

Figure 16. LaACES flight beacon. 


\section{Science Analysis \& Highlights of Some LaACES Team Results}

After recovery and a celebratory luncheon, the students spend the rest of the day working on their science presentations. Each group is given the GPS data along with timestamps collected by one of the GPS units so that they can relate their data with altitude. Wednesday is spent on preparing and finalizing science presentations. The students present their scientific results Thursday morning before all LaACES students and instructors as well as any CSBF employees who wish to attend. It is quite common for the presentations to be delivered to a packed house of CSBF personnel.

Some of the various types of experiments LaACES groups have implemented are still and video cameras to document the ascent and descent of the flight vehicle, environmental monitoring of pressure, temperature and relative humidity compared to NOAA predicted values, ozone measurements, atmospheric conductivity as a function of altitude, cosmic ray and neutron detectors, solar cell efficiency tests, measuring sound velocity versus altitude, UV measurements and magnetic field strength.

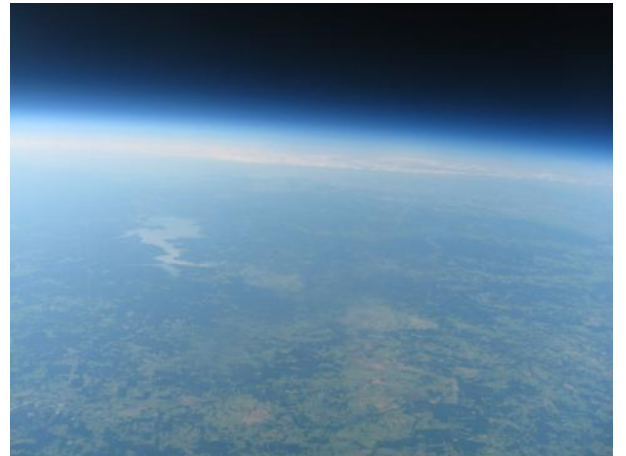

Figure 17. ACES-12: Onboard Camera.

During the 2006-2007 LaACES campaign, the McNeese LaACES Group Sound Experiment payload which flew on the ACES-06 flight sought to record environmental noise and measure sound velocity in relation to altitude.

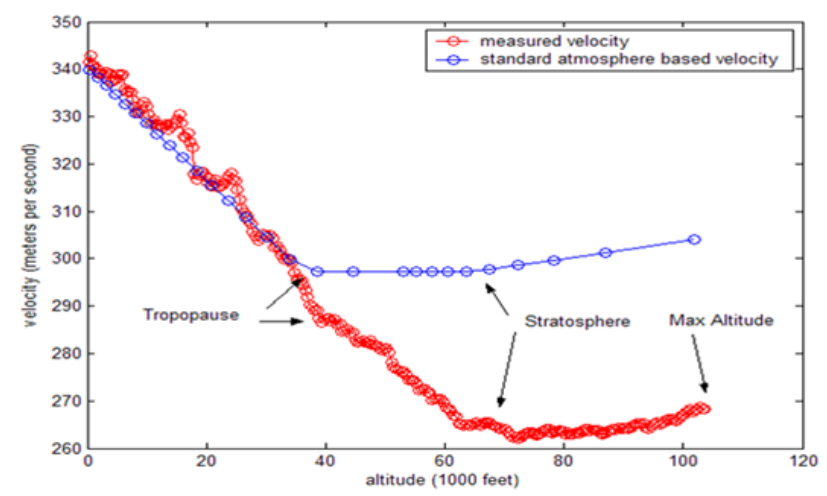

Figure 18. 2006-2007 LaACES campaign, results from the MLAGSE team from McNeese University ${ }^{10}$
While the observed data did not match up with the expected results, the students did manage to collect good data during flight and formulate several reasons why the observed results were different than the predicted. The students were also able to look at the sound in the stratosphere and determine from the lack of interference that there is little to no wind activity within that layer of the atmosphere (Fig. 18).

Also during the 2006-2007 LaACES campaign, a team from the Mechanical Engineering department at LSU ran a solar cell efficiency experiment which flew on the ACES-07 flight. Their goal was to measure the maximum power output and efficiency from the solar cells over time and altitude. Not only did they determine the maximum efficiency and the altitude at which it occurred (11\%@11 km,) they also determined that their efficiency increased as temperature decreased (Fig.19).

\section{Conclusion}

Including the ACES pilot program, LaACES has seen nine successful campaign years during which time it has launched 12 balloons each with multiple payloads attached. Over 150 undergraduate students from seven different universities in Louisiana have participated in the program and the LaACES concept and materials have been exported to other states as well as into Canada. We have also built upon the success of LaACES by assisting minority serving institutions to develop their own student ballooning program (see 16, this conference) and, in partnership with the NASA Balloon Program Office, have developed the High Altitude Student Platform (HASP) ${ }^{12,13}$ that can carry up to twelve advanced student payloads to 120,000 feet for a period of $15-30$ hours (see 15 , this conference)

Another first for the LaACES program is cooperation between the LSU Electrical Engineering Department and the Physics Department to provide graduating seniors in Electrical Engineering a senior design or capstone project involving a high altitude balloon project. Several Electrical Engineering students teamed with the departments of Biology and Physics to design and prototype an experiment that will investigate the possible existence of organisms living at high altitudes. Already

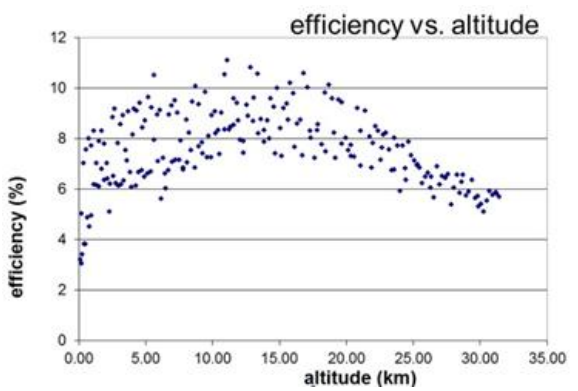

Figure 19. Results from the solar cell efficiency team from $2007^{11}$ 
several 2010-2011 LaACES students have shown interest in continuing this partnership next year.

LaACES students have been selected for NASA academies and NASA internships. Of those that have graduated, most have gone on to graduate school. Of those seeking employment, several have found jobs at industrial firms (e.g. Lockheed Martin) while others are in the application process. The LaACES experiences have a positive effect upon the students and is, we believe, helping to produce more capable students for STEM careers.

\section{Acknowledgments}

We thank the staff at the Columbia Scientific Balloon Facility for assistance with many balloon flights and Jenny Hay for help on the manuscript. This work was supported by LaSPACE under NASA Space Grant awards NNGO5GH22H and NNX10AI40H.

\section{References}

${ }^{1}$ Walker, R.S., Peters, F.W., Aldrin, B., et al., "Final Report of the Commission on the Future of the United States Aerospace Industry”, Crystal Gateway 1, Suite 940, 1235 Jefferson Davis Highway, Arlington, Virginia 22202, 2002, http://ita.doc.gov/td/aerospace/aerospacecommission/aerospacecommission.htm

${ }^{2}$ Dawson, J., "Science Board Warns of Uncertain Future for US Science and Engineering Leadership", Issues and Events, Physics Today, 57, No. 7, 25, 2004

${ }^{3}$ National Science Board, "Science and Engineering Indicators 2004", Two volumes, Arlington, VA: National Science Foundation (volume 1, NSB 04-1; volume 2, NSB 04-1A), 2004

4"The STEM Workforce Challenge: the Role of the Public Workforce System in a National Solution for a Competitive Science, Technology, Engineering, and Mathematics (STEM) Workforce", report prepared for the U.S. Department of Labor, Employment and Training Administration by Jobs for the Future, April 2007, http://www.doleta.gov/youth_services/pdf/STEM_Report_4\%2007.pdf

${ }^{5}$ Hart, G., Rudman, W. B., Armstrong, A., et al., "Road Map for National Security: Imperative for Change", The Phase III Report of the U.S. Commission on National Security / $21^{\text {st }}$ Century, Washington, D.C., 2001, http://govinfo.library.unt.edu/nssg/index.html

${ }^{6}$ Giammanco, J., "SkeeterSat Assembly and Operation Manual", LaACES Student Ballooning Course, August 2004, http://laspace.lsu.edu/aces/BalloonCourse/index.php

${ }^{7}$ Guzik, G.., "Developing a Team Contract", LaACES Project Management Course, August 2004, http://laspace.lsu.edu/aces/BalloonCourse/index.php

${ }^{8}$ Blackburn, K., Burleigh, S., and Tran, J., "LaACES Program 2009-2010 Flight Readiness Review Document for the Electrical Conductivity Experiment", May 2010

${ }^{9}$ Blackburn, K, Burleigh, S., Tran, J., "LaACES Program 2009-2010 Flight Readiness Review Document for the Electrical Conductivity Experiemnt”, May 2010

${ }^{10}$ Graves, K., Katiwata, A., King, T., Shreat, S., Townsend, T., "Team MLAGSE Science Presentation”, May 2007, http://laspace.lsu.edu/aces/teams/2006-2007/McNeese/McNeese_1.php

${ }^{11}$ Bounds, T., Dunn, A., Sasser, J., "Team Spandex Science Presentation”, May 2011, http://laspace.lsu.edu/aces/teams/2006-2007/LSU-ME/LSUBR ME 2.php

${ }^{12}$ Guzik, T.G. "Advanced PACER on the High Altitude Student Platform Call for Applications", Louisiana Space Consortium, Louisiana State University, Baton Rouge, LA, 2011

${ }^{13}$ Guzik, T.G. et al.,'Development of the High Altitude Student Platform”, J. Adv. Space Res., 42, 1704-1714, 2008

${ }^{14}$ Ellison, B., Giammanco, J., Guzik, T.G., Johnson, K., and Wefel, J.P., “The Louisiana ACES student-built BalloonSat program”, Advances in Space Research, 38, 2253-2258 (2006)

${ }^{15}$ Guzik, T.G., Ellison, S.B., Stewart, M., Wefel, J.P., Pierce, D. and Garde, G. “A Multiple Payload Carrier for High Altitude Ballooning", $2^{\text {nd }}$ Annual Academic High Altitude Conference, (This conference), Iowa State University, Ames, Iowa, June 22-24, 2011.

${ }^{16}$ Guzik, T.G., Browne, D, Ellison, S.B., Giammanco, J., Granger, D., Stewart, M., and Wefel, "Developing Student Ballooning Research Programs at Minority Institutions", $2^{\text {nd }}$ Annual Academic High Altitude Conference, (This conference), Iowa State University, Ames, Iowa, June 22-24, 2011.

${ }^{17}$ Spring, A., Dupris, R., Freeman, W., Gardiner, H., Myers, C. and Guzik, T.G., "The Pressure, Humidity, And Temperature - Test and Camera Observations (PHAT-TACO) Student-Built Balloon Payload", $2^{\text {nd }}$ Annual Academic High Altitude Conference, (This conference), Iowa State University, Ames, Iowa, June 22-24, 2011. 